\title{
Donor age significantly influences the Raman spectroscopic biomolecular fingerprint of human pancreatic extracellular matrix proteins following collagenase-based digestion
}

Rebecca M. Spiers ${ }^{a}$, Julia Marzi ${ }^{\text {b, d }}$, Eva M. Brauchle ${ }^{\text {b,d }}$, Sarah E. Cross ${ }^{a}$, Rebecca H. Vaughan $^{a}$, Paul A. Bateman ${ }^{a}$, Stephen J. Hughes ${ }^{a}$, Katja Schenke-Layland ${ }^{\text {b,c,d }}$, Paul R. V. Johnson $^{\mathrm{a}}$

a Islet Transplant Research Group, Nuffield Department of Surgical Sciences, University of Oxford, Level 6, John Radcliffe Hospital, Oxford, OX3 9DU and Oxford Centre for Diabetes, Endocrinology and Metabolism (OCDEM), University of Oxford, Churchill Hospital, Oxford, OX3 7LE, UK.

${ }^{b}$ Department of Women's Health, Research Institute for Women's Health, Eberhard-KarlsUniversity Tübingen, Silcherstr. 7/1, 72076 Tübingen, Germany

c Department of Medicine/ Cardiology, Cardiovascular Research Laboratories, David Geffen School of Medicine at UCLA, 675 Charles E. Young Drive South, MRL 3645, 90095 Los Angeles, CA, USA

${ }^{d}$ Natural and Medical Sciences Institute (NMI) at the University of Tübingen, 72770 Reutlingen, Germany

Corresponding author: Rebecca M Spiers, Islet Transplant Research Group, Nuffield Department of Surgical Sciences, University of Oxford, Level 6, John Radcliffe Hospital, Oxford, OX3 9DU, UK. Tel: +44 (0)1865 857507, Email: rebecca.spiers@nds.ox.ac.uk 


\section{Abstract}

Despite the enormous advances in the field of clinical pancreatic islet transplantation over the past two decades, the human islet isolation procedure remains suboptimal. Islets are extracted (isolated) from the exocrine tissue of donor pancreases using neutral protease (NP) and collagenase-based enzymes, which digest the extracellular matrix (ECM) scaffold surrounding human islets. This process remains highly variable and current isolation enzyme blends are ineffective at digesting pancreases from younger donors with low body mass indexes (BMI). However, age-related differences in pancreatic matrix digestion have not been studied in detail at the molecular level. To address this, we investigated ECM digestion in purified ECM proteins and in pancreatic tissue sections from younger ( $\leq 30$ years; $n=5$ ) and older ( $>55$ years; n=5) BMI matched donors, using Raman microspectroscopy (RMS). The Raman spectral profiles for purified collagens I, IV, VI and laminins were significantly altered following controlled enzyme treatment. Pancreatic cryosections were treated with Serva collagenase, NP, or the two enzymes combined, at clinically relevant concentrations. RMS demonstrated that the ECM at the islet-exocrine interface was differentially digested with respect to donor age. The action of collagenase was affected to a greater extent than NP.

RMS is a powerful, marker-independent technology for characterising the human pancreatic ECM and demonstrating differences between donor types. Ongoing detailed studies using RMS will assist the development of donor-specific enzyme blends, increasing the overall success of human islet isolation and benefiting many people with type 1 diabetes worldwide.

Keywords (Max 5): Islet transplantation; Islet isolation; Extracellular matrix; Collagenase; Raman microspectroscopy 
Abbreviations:

T1DM; Type 1 Diabetes Mellitus

ECM; Extracellular matrix

PCA; Principle component analysis

PC; Principle component

PIC; Protease Inhibitor Cocktail

NP; Neutral protease

RMS; Raman microspectroscopy

TCA; True component analysis

HBSS; Hanks buffered saline solution

DMCU; Dimethylcasein unit

Peri-islet; Islet periphery

r.t.; Room temperature

PBS; Phosphate buffered saline

BM; Basement membrane

IM; Interstitial matrix 


\section{Introduction}

Clinical pancreatic islet transplantation is a minimally invasive treatment that has the potential to reverse Type 1 Diabetes Mellitus (T1DM) in selected patients. It has widely been demonstrated that this procedure can significantly improve glycaemic control in patients with T1DM [1-3], and in particular, resolve life-threatening hypoglycaemic unawareness [4]. The islets of Langerhans comprise the $2 \%$ endocrine component of the pancreas, and prior to transplantation, they must be successfully extracted from the surrounding exocrine tissue during the critical islet isolation procedure. Once isolated, they are then transplanted by infusion into the recipient's liver using minimally-invasive radiological techniques [5]. Although the clinical outcomes of islet transplantation continue to improve, its wider implementation as a mainstay treatment option for T1DM is partly hindered by the variability of the human islet isolation procedure, where transplantable islet yields are obtained from less than $50 \%$ of pancreases processed worldwide [6]. In addition, in order to maintain adequate long-term glycaemic control, recipients often require more than one islet infusion from different pancreases [7].

The unpredictability of human islet isolation largely results from the variability of the enzymatic digestion stage of the isolation procedure. This is partly due to the inconsistency in composition of the different batches of commercially available collagenases that are used in human islet isolation [8]. In addition, donor factors are known to have a major influence on the efficiency of the islet extraction process. Digestion of the human pancreas is undertaken by delivering collagenase and protease-based blends through the ductal network, where the enzymes penetrate the islet-exocrine interface. Here they digest the extracellular matrix (ECM) within this region, to liberate exocrine free islets for subsequent density-gradient purification and transplantation [6]. Donor age is one of the most commonly reported factors known to influence isolation outcomes [9-13]. This results from the high percentage of islets that remain trapped (i.e. surrounded) by exocrine tissue following collagenase digestion in 
younger donors, which negatively influences post-purification yields $[14,15]$. As current isolation protocols are effective in extracting islets from older donors, poor digestion outcomes likely result from age-related compositional changes in the pancreatic ECM. This renders the standard isolation enzyme blends largely ineffective when applied to younger donor substrates. However, this presumption has never been investigated in a detailed manner at the molecular level.

The human pancreatic ECM is made up of a uniquely composed duplex basement membrane (BM), in addition to an adjacently connected interstitial matrix (IM). In the BM, Collagen IV selfassembles to form an expansive scaffold, which integrates with laminin glycoproteins and heparan sulphate proteoglycans, to form a highly organised supramolecular architecture [16]. Adjacently connected IM proteins include collagen types I, III, V and VI [17, 18]. As well as bordering the peripheral (peri) islet exterior, a distinct ECM is present within the intra-islet region, which acts to support the blood vessels located within the islet interior [19].

Although the impact of age on the structure and composition of the pancreatic ECM has been studied previously $[18,20-22]$, little is known about the effect of age on the digestion of these proteins during human islet isolation. By utilising an in-house developed glass slide based digestion assay, we have recently shown that donor age significantly affected the digestion of collagen IV and the $\alpha 5$ chain of laminin, when pancreatic tissue sections were treated with islet isolation enzymes at clinically relevant concentrations [23]. When utilising specific enzyme components, digestion efficiency of both proteins was significantly poorer in younger donors, providing evidence that donor age affects ECM digestion within the human pancreas [23].

To date, the majority of knowledge regarding the structure and composition of the pancreatic ECM has been generated from studies utilising conventional immunolabelling [18, 19, 22-24]. Although this research has provided insight into the unique spatial distribution of the specific 
ECM proteins within the islet region [19], identification has been limited to a pre-selected subset of proteins. In addition, when using immunolabelling to assess protein digestion, it is assumed that there is uniformity between the digestion of the regional epitopes recognised by the antibodies, and the rest of the protein molecule. Recent studies employing sophisticated high-throughput proteomics have enabled the comprehensive characterisation of the pancreatic matrisome, but at the cost of spatial resolution [25]. This highlights the requirement for a marker-independent microscopic technology, which is capable of characterising the ECM at the islet-exocrine interface, whilst avoiding any pre-selected user bias.

Raman microspectroscopy (RMS) is a non-contact, marker-independent laser-based technology that has gained increasing interest in the field of biomedical research. Following interaction with a sample, incident monochromatic light is scattered, with the resulting frequency shift directly related to molecularly specific vibrations within the tissue. RMS allows the identification of molecular groups and compositions in complex samples, with spectra generated from specific morphological tissue regions through the coupling of a conventional microscope $[26,27]$. RMS has been used to characterise ECM structures in various tissues, which has allowed the non-destructive monitoring of the pathological status of tissues and individual cells [26-28]. Next generation Raman instruments are capable of incorporating powerful spectroscopic imaging functions. Multivariate analysis tools are used to map biochemical constituents of a defined sample area onto a compositional image. Raman spectral information can be collectively transferred into a false colour code. Resulting spectroscopic images have good spatial resolution, allowing the localisation of proteins within complex tissues to be explored without utilising labels $[29,30]$. It is possible to use RMS to monitor the collagenase induced digestion of the collagen networks within tissues, with enzyme treatment resulting in the loss of Raman signal across the whole collagen spectra [27]. Specific collagen subtypes and changes in collagen structures can be identified on the basis of subtle spectral variations $[27,31]$. 
The main aim of this study was to use RMS to characterise the impact of donor age on the enzymatic digestion of the human pancreatic ECM, with the purpose to ultimately improve the clinical outcomes of human islet isolation. To do this, we (i) identified the Raman spectra for selected ECM proteins, by obtaining reference measurements from purified collagens I, IV, VI and laminins, which are prevalent in the human pancreas, (ii) performed a series of digestion experiments on these purified ECM proteins to demonstrate that RMS is capable of detecting ECM protein digestion, and (iii) identified the ECM spectra of human pancreatic tissues, and obtained Raman measurements from the tissue following enzyme treatment, to demonstrate the ability of RMS to detect donor-age related differences in ECM digestion. In addition, we explored using high resolution Raman imaging to obtain detailed marker-independent images of the islet and the surrounding ECM based on the sample's Raman spectra.

\section{Materials and Methods}

\subsection{ECM reference standards and digestion}

Purified human collagen IV (C533-5mg, $0.7 \mathrm{mg} / \mathrm{ml})$ and laminins (L6274-5mg, $0.5 \mathrm{mg} / \mathrm{ml})$ were purchased from Sigma (Gillingham, UK). Collagens I and VI (Ab7533 and Ab7538, both 1 $\mathrm{mg} / \mathrm{ml}$ ) were purchased from Abcam (Cambridge, UK). For proof of principle experiments, purified proteins were digested using collagenase NB1 and neutral protease NB (NP) (both Serva, Heidelberg, Germany) at concentrations relevant for human islet isolation (collagenase 7.2 PZU/ml, NP 0.14 dimethylcasein units (DMCU)/ml). $50 \mu \mathrm{g}$ of each reference standard were added at a 1:2 volume ratio to the enzyme solution, and heated at $37^{\circ} \mathrm{C}$ for 30 minutes, 120 minutes, or overnight. For control samples, the enzyme solution was replaced with HBSS (Lonza, Basel, Switzerland). At the end of the incubation period, the enzyme reaction was ceased by cooling to $4^{\circ} \mathrm{C}$ with the addition of protease inhibitor cocktail to all samples, including controls (PIC; cOmplete ${ }^{\mathrm{TM}}$ Mini Protease Inhibitor Cocktail, Roche). Samples were stored at $-20^{\circ} \mathrm{C}$ until use. 


\subsection{Human tissue sampling and preparation of cryosections}

This study was approved by our institutional review board South Central - Oxford Research Ethics Committee (NHS Health Research Authority), reference number 09/H0605/2. Written informed consent was obtained from donor relatives.

Human pancreases from older ( $>55$ years old; $n=5)$ and younger ( $\leq 30$ years old; $n=5)$ multiorgan donors were procured for islet isolation and received at the Oxford DRWF Human Islet Isolation Facility (Table 1). For all donors, donation was made following brain death certification (i.e. donation after brain death; DBD). $0.5 \mathrm{~cm}^{3}$ tissue samples were taken from the head of the pancreas and snap frozen in liquid nitrogen. Frozen tissue sections $(10 \mu \mathrm{m}$ thickness) were prepared using a Cryostat (Microm HM 560 cryotome, Thermo Scientific, Loughborough, UK) and collected onto silanized coverslips (WWR, Leicestershire, UK). Tissue sections were stored at $-20^{\circ} \mathrm{C}$ until use.

\subsection{Digestion of pancreatic tissue sections and immunofluorescent labelling}

Pancreas cryosections were thawed in HBSS (Lonza) and incubated for 5 minutes at $37^{\circ} \mathrm{C}$ with HBSS (control), collagenase alone (7.2 PZU/ml), NP alone (0.14 DMCU/ml; both Serva) or the two enzymes combined. Following treatment, enzymatic activity was quenched by placing coverslips in cold running water. Sections were fixed with $2.5 \%$ paraformaldehyde (Sigma) and then blocked with 5\% normal swine serum (Vector Laboratories, Peterborough, UK). Sections then underwent double immunofluorescent labelling. Incubation with primary antibodies: polyclonal guinea pig anti-insulin (1:100; A0564, Dako, Ely, UK), and polyclonal goat anti-collagen IV (1:300; 134001, BioRad, Oxford, UK) was for 1.5 hours at room temperature (r.t.). Sections were then incubated with secondary antibodies for 30 minutes at r.t: AlexaFluor 594 conjugated donkey anti-guinea pig (1:500; 706-585-178, Jackson Immunoresearch, PA, USA) and AlexaFluor 488 conjugated donkey anti-goat (1:500; A11055, Life Technologies). Post removal of the secondary antibodies, the coverslips were immersed in PBS and stored at $4^{\circ} \mathrm{C}$ prior to the Raman measurements. 


\subsection{Raman measurements}

\subsubsection{Purified protein reference standards and enzyme-treated proteins}

For measurement of digested and untreated purified proteins, a customised WITec alpha 300R Raman microscope was used (WITec GmbH, Ulm, Germany). A green laser (532 nm) and a spectrograph with a grating of $600 \mathrm{~g} / \mathrm{mm}$ were chosen for spectral detection. $20 \mu \mathrm{l}$ of both the enzyme-treated and control samples for collagens I, IV, VI and laminins were thawed and air dried overnight in separate glass bottom dishes (ibidi, Martinsried, Germany). For each protein, 45 measurements were obtained per condition, with a laser power of $50 \mathrm{~mW}$ and an acquisition time of 0.5 seconds per spectrum. The enzyme and PIC additives were measured in isolation. Single point Raman measurements of 10 acquisitions of 1 second each were also obtained from the purified proteins prior to incubation, to obtain spectra that were free from these additives.

\subsubsection{Measurements from native human tissue}

The WITec Raman system with a $532 \mathrm{~nm}$ green laser was used to identify the ECM spectra of human pancreatic tissue. Non-digested, rehydrated and Collagen IVIAlexaFluor 488 stained sections of pancreatic tissue were measured with a 63x Apochromat water dipping objective (N.A. 1.0; Carl Zeiss GmbH, Jena, Germany). Islets were identified according to the immunofluorescence staining and an area of $200 \times 200 \mu \mathrm{m}$ was imaged with a scanning step size of $2 \mu \mathrm{m}$. The laser power was set to $50 \mathrm{~mW}$ and an acquisition time of 0.5 seconds was used per spectrum. Four donors (two from each age group) were selected for the Raman imaging experiments, with two islets imaged per donor. In addition, higher resolution images at a pixel resolution of $1 \times 1 \mu \mathrm{m}$ and an area of $100 \times 100 \mu \mathrm{m}$ were acquired.

\subsubsection{Measurements from digested human tissue}

RMS was performed for single spectra acquisition using a custom set-up Raman microspectroscope as described previously $[32,33]$ coupled to a fluorescence microscope 
(Olympus, Hamburg, Germany). Briefly, the Raman spectrometer was equipped with a 784 nm diode laser (Toptica Photonics, Munich, Germany), which induced Raman scattering when directed at the sample through the microscopic objective (60x water immersion, NA 1.2, Olympus). As Raman shifts represent a wavenumber shift relative to the applied laser wavelength, it allows for comparative analysis of data that were acquired at different laser wavelengths (i.e. 532 and $784 \mathrm{~nm}$ ). Raman spectra were detected using an air-cooled charged couple device (CCD) camera (Andor iDus). Single point Raman tissue measurements were acquired from treated tissue sections, fully immersed in 1X PBS. For all donors ( $n=5$ for each age group), at least two islets were measured for each treatment (i.e. the control and all three enzyme treatments). Islets were identified with the microscope camera on the basis of positive insulin labelling, controlled by CellB software (Olympus). For each islet 15 Raman spectra were taken from islet peripheral and 5 from the intra-islet region. All measurements were restricted to collagen IV positive regions. Spectra were acquired with an integration time of 100 seconds, and a laser output power of $85 \mathrm{~mW}$ was used.

\subsection{Spectral analysis}

Raman images of the native pancreatic tissue were analysed by applying the Project Five 5.1 software (WITec $\mathrm{GmbH}$ ), using true component analysis (TCA). The principles of TCA have been explained in detail before [34]. Briefly, TCA is a multivariate analysis tool that defines the most prevalent spectral components in a data set. One component describes pixels of similar spectral information, which can be projected onto an intensity distribution heat map ('Raman image'). Analysis of component spectra enables the identification of the respective tissue structure.

For the spectra obtained from pancreatic tissue using the custom Raman set up, processing was performed in OPUS (Bruker Optik GmbH, Ettlingen, Germany). Spectra were obtained from the cover glass and PBS alone after every tenth measurement and subtracted from the sample spectra. Baseline correction (64-point rubberband method) was then performed. To 
ensure that spectral differences could be attributed to the enzyme treatment, all single-point spectral measurements were normalised to the corresponding control spectra for each donor. This was achieved by averaging the control spectra to the value of 1 , before dividing each equivalent spectral position in the enzyme treated spectra by this modified control. Data normalisation was performed in Excel (Microsoft Corporation, USA, 2016). Finally, the spectra were cropped in the region of $480-1680 \mathrm{~cm}^{-1}$.

Due to complex molecular patterns of ECM structures, multivariate analysis such as principal component analysis (PCA) is required to resolve complex molecular shifts [33]. PCA is commonly implemented to determine differences and similarities in chemometrics. The principles of PCA for the analysis of Raman data have been explained in detail before [32, 35]. Collectively, the devised principle components (PCs) describe the total information contained within the original data set. The first PC explains the highest amount of variation, and the subsequent PCs explain the next highest amounts of variation chronologically. PCA results are interpreted on a scores plot, where one PC is graphed against another, with every spectrum represented by an individual dot. Whilst similar spectra cluster together on the scores plot, dissimilar spectra are separated. The corresponding PC loading plot displays the contributions of each spectral region to the overall structure of the scores plot. PCA was completed in Unscrambler X (CAMO Software, Norway). Data were vector-normalised and smoothed using Savitzky-Golay filtering. The PCs that were most effective at separating the data from different experimental groups were selected, and their subsequent loading spectra were examined. PCA was used to analyse both the digested purified protein experiments and the enzyme treated pancreatic tissue experiments.

\subsection{Statistical analysis}

Data are presented as mean \pm standard deviation (SD). Data normality was determined by Shapiro Wilk test. Statistical significance of PCA scores was determined by Mann-Whitney U. 
Statistical analyses were performed in Prism/GraphPad (USA). Data with $p$-values of less than 0.05 were identified as statistically significant.

\section{Results}

\subsection{Raman spectra for purified matrix proteins}

To determine if specific matrix proteins could be distinguished on the basis of their Raman spectra, we first obtained Raman measurements from human purified collagens, I, IV, VI and laminins. These proteins were selected based on their prevalence in the human pancreas [17, 18]. We also demonstrated the distribution of these proteins in the pancreas, using immunofluorescent labelling. All 4 proteins were present throughout the exocrine pancreas, in addition to being present at the islet-exocrine interface (Supplementary Figure 1). Figure $1 \mathrm{~A}$ displays the Raman reference spectra following baseline correction and normalisation. Anticipated characteristic protein Raman bands were present, such as: proline $\left(854-858 \mathrm{~cm}^{-}\right.$

$\left.{ }^{1}\right)$, C-C backbone stretching $\left(938 \mathrm{~cm}^{-1}\right)$, phenylalanine $\left(1005 \mathrm{~cm}^{-1}\right)$ amide III $\left(1242 \mathrm{~cm}^{-1}\right), \mathrm{CH}_{2}$ deformation (1449-1465 $\left.\mathrm{cm}^{-1}\right)$ and amide I $\left(1666 \mathrm{~cm}^{-1}\right)$ [36-40]. Although the four proteins displayed a high degree of spectral homogeneity, subtle protein specific characteristics were present. This included shifting of feature peaks, such as proline (Figure 1 B) and $\mathrm{CH}_{2}$ deformations, where a distinctive double peak was noted for collagen $\mathrm{VI}\left(1453,1465 \mathrm{~cm}^{-1}\right)$ (Figure $1 \mathrm{C}$ ). Unlike the collagens, laminins lacked a clearly defined amide III band. Instead a peak was present at $1307 \mathrm{~cm}^{-1}$ (Figure $1 \mathrm{D}$ ).

\subsection{Changes of ECM fingerprint spectra due to enzymatic digestion}

We aimed to determine if RMS was capable of detecting characteristic changes in ECM spectra following enzyme treatment with clinical islet isolation blends over a time course. Purified matrix proteins were incubated with collagenase and neutral protease (NP) at enzyme concentrations clinically relevant for human islet isolation. Reactions were stopped by the addition of a protease inhibitor cocktail (PIC). Protein degradation in response to enzyme 
treatment was assessed by Raman spectral changes, when compared to a control, using principle component analysis (PCA). Control samples were heated overnight, and the enzyme replaced with HBSS. Spectra obtained from the PIC and enzyme alone revealed major peaks at $880 \mathrm{~cm}^{-1}$ (PIC) and $1047 \mathrm{~cm}^{-1}$ (enzyme) (Supplementary materials; Figure $2 \mathrm{~A}-\mathrm{B}$ ). These peaks were disregarded for the purpose of the analysis.

Enzyme treatment affected the overall composition of the Raman spectra obtained from all four ECM proteins, determined on the basis of separated spectral clustering's on the PCA scores plots. Collagen I separation occurred along the scores plot for PC-3, with the 120 minute and overnight treatment spectra migrating to the negative region of the PC (Figure 2 A). The PC loading displayed Raman bands including $940 \mathrm{~cm}^{-1}(\mathrm{C}-\mathrm{C}$ backbone stretching) and $1453 \mathrm{~cm}^{-1}\left(\mathrm{CH}_{2}\right.$ deformation) [40] (Figure $\left.2 \mathrm{~B}\right)$. For collagen IV, the 120 minute and overnight treatment spectra migrated towards the negative region of PC-2 (Figure $2 \mathrm{C}$ ). Peaks were present at $963 \mathrm{~cm}^{-1}$ (general protein assignment) [38] and $1453 \mathrm{~cm}^{-1}$ on the loadings for PC-2 (Figure $2 \mathrm{D}$ ). Separation of the control and enzyme spectra for Collagen VI was apparent in PC-1 (Figure $2 \mathrm{E}$ ). The overnight treated spectra formed a clear population in the negative region of the scores plot. The loading detected feature peaks including $940 \mathrm{~cm}^{-1}$ and $1453 \mathrm{~cm}^{-}$

${ }^{1}$ (Figure $2 \mathrm{~F}$ ). Spectral separation following enzyme incubation of laminins also occurred along PC-1, with the enzyme treated spectra clustering toward the negative region of the PC (Figure $2 \mathrm{G})$. The characteristic loading pattern for laminins was substantially different from the collagens. Prominent peaks were present at $761 \mathrm{~cm}^{-1}$ and $1469 \mathrm{~cm}^{-1}$, which appear to be previously unassigned in the literature (Figure $2 \mathrm{H}$ ).

\subsection{Identification of the ECM spectra in human pancreatic tissue}

Raman imaging was used to identify the ECM spectra in human pancreatic tissue. A spectral map was generated from a defined area of tissue, containing an islet. Islets were identified on the basis of the collagen IV labelling pattern. Every pixel within this area corresponded to a single Raman spectrum. Multivariate image analysis was performed to identify pixels that 
contain spectral information from biochemically similar structures. Each structure can be identified by a specific component spectrum. Intensities for each spectral component are then shown within the image. Three major spectral components were identified in overview scans of the pancreatic tissue (Figure 3) and their peak assignments were evaluated to assess their corresponding molecular structures (Table 2). Component 1 described the cytoplasm, containing peaks assigned to proteins and lipids such as 1081, 1123 and $1299 \mathrm{~cm}^{-1}$. Component 2 represented the ECM and displayed peaks at 854, 938 and $1245 \mathrm{~cm}^{-1}$ that related to the Raman scattering of defined molecular bonds in collagens such as vibrations of proline, C-C backbone and amide III. Component 3 correlated to nuclei and showed peaks at 784, 1098 and $1577 \mathrm{~cm}^{-1}$ referring to DNA, phosphate backbone and nucleic acid bases. In addition, there was a high degree of homogeneity between the Collagen IVIAlexaFluor488 specific signature (Figure 4 A-C) and the Raman identified ECM component (i.e. component 2) (Figure 4 D-F), which was observed when merging the images (Figure 4 G-I).

\subsection{Raman detects age-related differences in the digestion of the pancreatic ECM}

To induce digestion of the ECM, human pancreatic tissue sections from different aged donors were treated with the enzyme components found in human clinical islet isolation blends: collagenase, NP or the two enzymes in combination. The regional islet-exocrine interface was located by labelling for insulin and collagen IV (Figure $5 \mathrm{~A}$ ). Following enzyme treatment there was a visible loss of collagen IV signal for both younger and older donors, as compared to the untreated controls. Protein loss was evident in both the peri-islet matrix, and from the matrix surrounding the blood vessels in the intra-islet region (Figure 5 B). We have previously semiquantified the loss of this collagen IV staining morphometrically, following 5 minutes of enzyme treatment, and demonstrated that collagen IV is significantly lost during this period [23]. The collagen IV staining was used to obtain targeted single point Raman measurements (Figure 5 B). Spectra from enzyme-treated sections were normalised to those obtained from control tissue, to enable the investigation of spectral differences that resulted from the administered 
enzyme treatment. Digestion efficiency was not affected by the matrix location (i.e. peri-islet or intra-islet) and thus measurements were combined (Supplementary materials; Figure 3 AC). RMS successfully revealed age related differences in enzyme induced ECM degradation, which was demonstrated through changes in spectral regions corresponding to ECM proteins (Figure 6). Treatment of pancreatic tissue sections with collagenase alone resulted in spectral changes that were significantly different across the two age groups. These differences were reflected in the clear cluster separation of the two groups, in the scores displayed for PC-5 $(p<0.001$ for younger vs older PC-5 scores; Figure 6 A). The corresponding loading spectra included peaks at $758 \mathrm{~cm}^{-1}$ (unassigned peak), $1066 \mathrm{~cm}^{-1}$ (proline/saccharides), and $1296 \mathrm{~cm}^{-}$ ${ }^{1}\left(\mathrm{CH}_{2}\right.$ deformation) [36, 40, 41] (Figure $\left.6 \mathrm{~B}\right)$.

Treatment of tissue sections with NP alone resulted in fewer age-related variations in the overall spectral composition. Although the cluster separation between the age groups was less pronounced, it was still significant ( $p<0.05$ for younger vs older PC-5 scores; Figure 6 C). The corresponding PC-5 loading spectra identified peaks at $945 \mathrm{~cm}^{-1}$ (C-C backbone stretching) and $1444 \mathrm{~cm}^{-1}\left(\mathrm{CH}_{2}\right.$ deformation) [40] (Figure 6 D).

Combining collagenase with NP produced an age-related separation similar to that seen with collagenase treatment alone. The clustering was observed on the scores for PC-4 with the older donors centring towards the negative region of the scores plot $(p<0.001$ for younger vs older PC-4 scores; Figure 6 E). The peaks featured on the loading spectra reflected a combination of those that were present in the single enzyme treated loadings, including: 945 $\mathrm{cm}^{-1}, 1066 \mathrm{~cm}^{-1}, 1296 \mathrm{~cm}^{-1}\left(\mathrm{CH}_{2}\right.$ deformation) and $1444 \mathrm{~cm}^{-1}$ [40, 41] (Figure $6 \mathrm{~F}$ ).

\subsection{High-resolution Raman imaging enables marker-independent visualisation and characterisation of pancreatic ECM}

We investigated the value of obtaining higher resolution Raman images from the pancreatic tissue, for completing in depth compositional assessments of the pancreatic ECM. The enhanced resolution Raman images (pixel size $1 \times 1 \mu \mathrm{m}$ ) were obtained from tissue regions 
that contained an islet, identified by collagen IV labelling (Figure $7 \mathrm{~A}, \mathrm{~B}$ ). This enabled the identification of an additional matrix-related component, shown in orange (Figure $7 \mathrm{C}, \mathrm{D}$ ). Although the second matrix component displayed some co-localisation with the first, the two components were clearly morphologically distinct. Whilst the location of the second component was not restricted to the intra-islet region, its intensity within this region was higher than the first component. The representative spectra displayed several variations (Figure $7 \mathrm{E}$ ). The presence of two spectrally distinct matrix components prompted the detailed characterisation of the matrix within different regions of the islet. This was performed by extracting single spectra from the high resolution images. Spectra were extracted from the peri-islet and intra-islet ECM, on the basis of the corresponding collagen IV staining (Figure 8 A). The spectra obtained from these two regions were analysed by PCA. The scores plot of the PCA identified a highly significant difference in PC-3 score values when comparing periand intra- islet ECM spectra (Figure $8 \mathrm{~B}$ ). The loadings plot (Figure $8 \mathrm{C}$ ) indicated the most relevant spectral differences between these two distinct matrix regions. Peaks at 997, 1205 and $1663 \mathrm{~cm}^{-1}$ were most prevalent in the intra-ECM structures clustering in the positive PC3 range. The observed Raman peaks correlated to a higher intensity of the amide I peak [40] and a different hydroxyproline-proline ratio referring to differences in collagen structures [26, 42]. The peri-islet ECM spectral data clustered in the negative $\mathrm{PC}-3$ range that was described by spectral assignments to peaks at 854,938 and $1242 \mathrm{~cm}^{-1}$. These peaks were also found in the collagen reference spectrum.

\section{Discussion}

Our study is the first to explore the feasibility of using RMS to monitor enzyme-induced digestion of the human pancreatic ECM from different aged donors. We also employed Raman imaging to obtain high quality biochemical images of the ECM surrounding human islets in a marker-independent manner. 
We obtained Raman spectra from the purified matrix proteins collagens I, IV, VI and laminins, to determine if Raman could resolve distinct matrix proteins on the basis of their biochemical fingerprints. For collagens, the overall spectral composition were similar to that described previously [27, 31]. Despite the high degree of homogeneity, subtle spectral variations existed, including a peak shift in the region corresponding to proline. In native collagen, proline often occupies one of the three amino acid positions in the tri-peptide repeat that represents the triple helical domain of collagen [43]. Therefore, peak shifts in this region likely result from structural differences in the collagenous domain between the different collagen subtypes. Although proline is present in laminins, its content is lower than in collagen [44]. A similar peak shift was observed in the region corresponding to general $\mathrm{CH}_{2}$ deformation in proteins.

Subsequently, in a series of proof of principle experiments, we treated purified proteins with the collagenase and NP blend utilised in the clinical isolation of human islets, to determine if Raman could detect post-treatment changes in protein structure. Protein treatment occurred over a time course, and with the exception of collagen IV incubation for 30 minutes, all protein treatment spectra were significantly different from the respective controls. In the collagens, spectral regions affected by enzyme treatment corresponded with $\mathrm{CH}_{2}$ deformations (1453 $\mathrm{cm}^{-1}$ ) and C-C stretching $\left(940 \mathrm{~cm}^{-1}\right)$, which represented a general change in collagen composition due to digestion. The subtle shifts in the original peak positions were attributed to the enzyme treatment. Interestingly, post-enzyme treatment, the characteristic changes in the spectral profile of laminins varied drastically from the three collagens. Although this probably reflects differences in the native protein structure, it may also represent key differences in the action of specific enzyme components, as the digestion of laminins, unlike collagen, is known to be mediated by NP $[23,45]$.

We used Raman imaging as a marker-independent approach to study the biochemical composition of the pancreatic ECM associated with human islets. To ensure that our results are clinically applicable, the tissue used in these experiments was exclusively obtained from 
pancreases that had been accepted for clinical islet isolation. This guaranteed that the organ ischemia time was tightly controlled, in addition to other confounding factors that would likely influence islet extraction and quality. The availability of clinical grade pancreases is limited, as is the opportunity to obtain biopsies from these organs for research. Although we acknowledge that this has restricted the number of donors that we have used in these studies, it is common practice of human pancreas and islet investigations of this nature to use an equivalent number of donors to those presented here $[18,23]$. Such sample numbers are also comparable to previously published studies that have used Raman spectroscopy for matrix characterisation $[26,27]$. Within the pancreatic tissue we successfully identified a component that defined nuclei and the cytoplasm, with the corresponding Raman spectra featuring characteristic DNA and protein/lipid peaks respectively. In addition, an ECM specific component was identified. The optical biochemical fingerprint of this component displayed a remarkable degree of homogeneity to the corresponding collagen IV specific immunostaining. After establishing the anticipated Raman spectra for ECM proteins in the human pancreas, we used RMS to study the effect of donor age on the enzyme induced digestion of the human pancreatic ECM. As previous studies have reported the importance of attenuating the ratio of collagenase and NP for enhancing islet isolation outcomes from younger donors $[22,46]$, we chose to study the action of both enzymes separately, as well as in combination.

Implementation of a unique spectral processing step, which involved normalising the spectra obtained from the enzyme treated tissues to the respective controls, ensured that differences in ECM biochemical composition following enzyme treatment were attributable to age-related variations in enzyme action. Variations in peak positions between the reference standards and the tissue can be accredited to the structural differences between purified proteins and those found in vivo [32]. Treatment of tissue sections with the blends containing collagenase resulted in the most pronounced age-related affects, though independent treatment with NP also resulted in significant differences in protein digestion between the two age groups. These results suggest that age-related differences in pancreatic ECM digestion are more dependent 
on collagenase action, than on NP. Therefore, this difference in collagenase activity may be the underlying reason for poor pancreas digestion from younger donor organs. Supporting our theory is a study from the Baylor group, which showed that doubling the concentration of collagenase when isolating islets from donors $<30$ years old results in a significantly lower proportion of trapped islets [46]. However, the enzyme comparisons were completed across different donor organs. Based on our RMS results, we are currently using a split lobe model to investigate how increasing collagenase concentrations may enhance isolation success from younger donors, whilst comparing for intra-pancreatic variability. Using a similar split pancreas model, researchers at the University of Louisville demonstrated that NP, but not collagenase, significantly reduced the number of trapped islets and enhanced post-purification islet recovery in donors aged $<35$ [22]. However, unlike in our study, measurement of age-related digestion-based differences did not occur at the molecular level.

The biological reasoning behind the demonstrated age-related digestion differences in matrix proteins has not been fully elucidated. However, it likely results from differences in matrix protein quantity between the two age groups. In support of this theory, we have recently used high-throughput proteomics to characterise the pancreatic matrix in younger and older donors. We identified that 19 matrix proteins were more highly expressed in younger donors, including 14 collagens (unpublished data). It is reasonable to assume that the increased matrix content in younger donors impacts on collagenase activity.

Investigations into the enzymatic digestion of the pancreatic ECM have previously been restricted to immunostaining methods. This has involved incubating small pancreatic tissue biopsies in various enzyme solutions, followed by cryosectioning and immunolabelling [45], or alternatively, the direct application of enzymes to sectioned tissue $[23,24,48]$. Loss of protein is in turn determined based on a reduction in the staining intensity of specific ECM proteins. Antibody based detection offers a straightforward approach for investigating specific protein molecules. However, when measuring the enzymatic breakdown of proteins, the assessment 
of digestion is confined to the regional epitope recognised by the antibody, and thus assumes uniform degradation between this region and the rest of the molecule. Indeed, although this can be somewhat overcome by utilising antibodies that identify multiple epitopes (i.e. polyclonal antibodies), Raman is capable of offering a label-free approach for monitoring protein digestion [27]. Immunostaining is utilised, but only for the purpose of directing the laser spot, with Raman measurements reflective of ubiquitously expressed molecular bonds within the protein [27]. This molecular based assessment arguably makes RMS a more robust method for monitoring protein digestion, which is not restricted by local antibody binding. Furthermore, our data substantiates the usefulness of using RMS for detecting donor related differences in the digestion of the islet matrix in the context of human islet isolation. However, it must be noted that although our slide-based digestion method is an excellent way of controlling for intra-pancreatic variability, by allowing multiple enzyme blends to be tested on any one pancreas, it does not mimic the clinically utilised intra-ductal infusion of collagenase based enzymes [47]. It should also be acknowledged that the use of cryosections of pancreatic tissue in this assay may lead to increased exposure of the ECM surrounding the islet to the enzyme solution, which may not occur in the actual human islet isolation procedure.

The main limitation of this study is that we were unable to monitor the digestion of specific ECM proteins. This was partly due to the high similarity of the spectra obtained from the reference standards, which restricted the identification of unique spectral components for each of the four reference proteins. Furthermore, the ECM networks within tissues are highly heterogeneous. In vivo the ECM is composed of a highly integrated structure, whereby multiple proteins are intercalated to form tight networks [16]. This makes it technically challenging to target single proteins, within the ECM by RMS. Indeed, using conventional histology, our group has previously shown that key matrix proteins are differentially digested during human islet isolation [24]. Although this highlights the importance of characterising the 
digestion of specific proteins, it is first important to characterise the effect of age on the combined matrix, as found in situ.

Utilising Raman images of a higher resolution, enabled the identification of a second matrix component (ECM 2) which displayed a different localisation pattern to the first (ECM 1, both Figure 7). The spectra of ECM 1 and ECM 2 are both assigned to collagens. The differences between the spectra may be linked to the 3D structural arrangement and the orientation of collagen fibers. The presence of an additional, compositionally distinct, matrix component encouraged the characterisation of the ECM within different regions of the islet. This analysis demonstrated that there was a significant compositional difference in the matrix surrounding the islet periphery to that present within the intra-islet region. Regional differences in the laminins composition of the human islet matrix have been reported previously, with distinct subtype arrangements identified in the peri-islet BM, and each layer of the duplex BM surrounding intra-islet blood vessels [19]. Where laminin $\alpha 5$ was ubiquitously expressed in all matrix locations, laminin $\alpha 4$ was only present in the intra-islet matrix and absent from the periislet matrix [19]. In this study we did not utilise the reference standards required to necessitate the detection of specific laminin subtypes. Therefore, although our data did not allow for regional differences in the subtype expression of laminins to be confirmed, we were clearly able to show that ECM related peaks were differentially expressed between the two matrix locations. This included spectral regions corresponding to C-C collagen background stretching and amide I, which were associated with the peri- and intra- islet ECM spectra respectively. Previously it has been shown that collagenase enters the islet interior following standard intraductal administration. Here it becomes localised to the ECM surrounding the islet vasculature [49]. Although sufficient cleavage of the peripheral ECM is a prerequisite for the successful extraction of islets from the exocrine tissue, digestion of the intra-islet framework is likely to result in islet fragmentation [49]. Degradation of the intra-islet matrix has been confirmed following human islet isolation, with islets displaying limited matrix recovery during 
routine islet culture [24]. Identifying matrix components that are specific to the peri- or intraislet ECM will aid the development of tailored enzyme blends optimised to necessitate cleavage of the islet from the exocrine, whilst preserving the integrity of the intra-islet ECM. Previous studies have used mass spectrometry (MS) based imaging to characterise the spatial distribution of matrix proteins from sections of human tissues [50]. MS Images are presented as protein heat maps, detailing the spatial distribution of specific proteins in relation to morphological features [50]. However, it is important to note that MS requires extensive sample preparation, which leads to the destruction of the sample. RMS therefore provides a non-invasive alternative, requiring substantially less preparation time.

In conclusion, our data demonstrate for the first time the feasibility of using RMS to study the impact of donor-related variables on the digestion of ECM proteins at the islet-exocrine interface. This is the first study to use RMS to investigate pancreas digestion. RMS clearly demonstrated that the ECM at the islet-exocrine interface is differentially digested with respect to donor age. Our results indicated that donor age affected the action of collagenase to a greater extent than NP. In addition, we described using Raman imaging to characterise the composition of the islet ECM in untreated pancreatic tissue. Using this method we were able to identify compositional differences in the matrix surrounding the islet periphery, as opposed to that surrounding the intra-islet vasculature. This has important implications for preventing islet fragmentation, by preserving the integrity of the intra-islet ECM during pancreas digestion. In contrast to conventional histology, Raman measurements are reflective of the molecular bonds within the tissue and tissue processing is considerably less time consuming. Furthermore, ECM characterisation is not limited to pre-selected proteins, and digestion assessment is not restricted to the protein regions that are recognised by antibodies. Our study shows that RMS is a powerful, label-free technology, which can be used to characterise the human pancreatic ECM from different donor types. This may ultimately aid in 
the development of targeted donor-specific enzyme blends, improving the overall efficiency of human islet isolation.

\section{Acknowledgments}

This work was carried out in the Nuffield Department of Surgical Sciences and the Oxford Centre for Diabetes, Endocrinology and Metabolism, both at the University of Oxford, and the AG Schenke-Layland, Research Institute of Women's Health in the Department of Women's Health at the University Tübingen. The authors would like to thank the members of the Oxford Human Islet Isolation Team for their excellent work in the isolation facility.

\section{Disclosures}

The authors declared no potential conflicts of interest with respect to the research, authorship, and/or publication of this article.

\section{Funding}

This work was financially supported by the Juvenile Diabetes Research Foundation (JDRF), the Diabetes Research and Wellness Foundation (DRWF) (both to P.R.V.J.), the European Union's Horizon 2020 research and innovation programme under the grant agreement number 645991-2, DRIVE (to K.S.-L. and P.R.V.J.), the Ministry of Science, Research and the Arts of Baden-Württemberg (33-729.55-3/214 and SI-BW 01222-91 (to K.S-L.)) and the National Institute for Health Research (NIHR) Oxford Biomedical Research Centre (BRC). The views expressed are those of the authors and not necessarily those of the NHS, the NIHR or the Department of Health. R.M.S. was the recipient of a Short-Term Travel Fellowship from the European Molecular Biology Organisation (EMBO). E.M.B. was supported by a postdoctoral fellowship from the Peter and Traudel Engelhorn Foundation. 


\section{Ethical Approval}

This study was approved by our institutional ethical review board - Oxford Research Ethics Committee (NHS Health Research Authority), reference number 09/H0605/2.

\section{Statement of Informed Consent}

Written informed consent was obtained from donor relatives.

\section{References}

[1] B.J. Hering, W.R. Clarke, N.D. Bridges, T.L. Eggerman, R. Alejandro, M.D. Bellin, K. Chaloner, C.W. Czarniecki, J.S. Goldstein, L.G. Hunsicker, D.B. Kaufman, O. Korsgren, C.P. Larsen, X. Luo, J.F. Markmann, A. Naji, J. Oberholzer, A.M. Posselt, M.R. Rickels, C. Ricordi, M.A. Robien, P.A. Senior, A.M. Shapiro, P.G. Stock, N.A. Turgeon, C. Clinical Islet Transplantation, Phase 3 Trial of Transplantation of Human Islets in Type 1 Diabetes Complicated by Severe Hypoglycemia, Diabetes Care 39(7) (2016) 1230-40.

[2] P.J. O'Connell, D.J. Holmes-Walker, D. Goodman, W.J. Hawthorne, T. Loudovaris, J.E. Gunton, H.E. Thomas, S.T. Grey, C.J. Drogemuller, G.M. Ward, D.J. Torpy, P.T. Coates, T.W. Kay, C. Australian Islet Transplant, Multicenter Australian trial of islet transplantation: improving accessibility and outcomes, Am J Transplant 13(7) (2013) 1850-8.

[3] S. Lablanche, S. Borot, A. Wojtusciszyn, F. Bayle, R. Tetaz, L. Badet, C. Thivolet, E. Morelon, L. Frimat, A. Penfornis, L. Kessler, C. Brault, C. Colin, I. Tauveron, D. Bosco, T. Berney, P.Y. Benhamou, G. Network, Five-Year Metabolic, Functional, and Safety Results of Patients With Type 1 Diabetes Transplanted With Allogenic Islets Within the Swiss-French GRAGIL Network, Diabetes Care 38(9) (2015) 1714-22.

[4] C.B. Leitao, T. Tharavanij, P. Cure, A. Pileggi, D.A. Baidal, C. Ricordi, R. Alejandro, Restoration of hypoglycemia awareness after islet transplantation, Diabetes Care 31(11) (2008) 2113-5.

[5] R.C. Gaba, R. Garcia-Roca, J. Oberholzer, Pancreatic islet cell transplantation: an update for interventional radiologists, J Vasc Interv Radiol 23(5) (2012) 583-94; quiz 594. [6] P.R. Johnson, K.E. Jones, Pancreatic islet transplantation, Semin Pediatr Surg 21(3) (2012) 272-80.

[7] A.M. Shapiro, J.R. Lakey, E.A. Ryan, G.S. Korbutt, E. Toth, G.L. Warnock, N.M. Kneteman, R.V. Rajotte, Islet transplantation in seven patients with type 1 diabetes mellitus using a glucocorticoid-free immunosuppressive regimen, N Engl J Med 343(4) (2000) 230-8. 
[8] M.J. Barnett, X. Zhai, D.F. LeGatt, S.B. Cheng, A.M. Shapiro, J.R. Lakey, Quantitative assessment of collagenase blends for human islet isolation, Transplantation 80(6) (2005) 723-8.

[9] J.S. Kaddis, J.S. Danobeitia, J.C. Niland, T. Stiller, L.A. Fernandez, Multicenter analysis of novel and established variables associated with successful human islet isolation outcomes, Am J Transplant 10(3) (2010) 646-56.

[10] J.R. Lakey, G.L. Warnock, R.V. Rajotte, M.E. Suarez-Alamazor, Z. Ao, A.M. Shapiro, N.M. Kneteman, Variables in organ donors that affect the recovery of human islets of Langerhans, Transplantation 61(7) (1996) 1047-53.

[11] R. Nano, B. Clissi, R. Melzi, G. Calori, P. Maffi, B. Antonioli, S. Marzorati, L. Aldrighetti, M. Freschi, T. Grochowiecki, C. Socci, A. Secchi, V. Di Carlo, E. Bonifacio, F. Bertuzzi, Islet isolation for allotransplantation: variables associated with successful islet yield and graft function, Diabetologia 48(5) (2005) 906-12.

[12] S.C. Hanley, S. Paraskevas, L. Rosenberg, Donor and isolation variables predicting human islet isolation success, Transplantation 85(7) (2008) 950-5.

[13] R.P. Meier, I. Sert, P. Morel, Y.D. Muller, S. Borot, L. Badet, C. Toso, D. Bosco, T. Berney, Islet of Langerhans isolation from pediatric and juvenile donor pancreases, Transpl Int 27(9) (2014) 949-55.

[14] A.N. Balamurugan, B. Naziruddin, A. Lockridge, M. Tiwari, G. Loganathan, M. Takita, S. Matsumoto, K. Papas, M. Trieger, H. Rainis, T. Kin, T.W. Kay, S. Wease, S. Messinger, C. Ricordi, R. Alejandro, J. Markmann, J. Kerr-Conti, M.R. Rickels, C. Liu, X. Zhang, P. Witkowski, A. Posselt, P. Maffi, A. Secchi, T. Berney, P.J. O'Connell, B.J. Hering, F.B. Barton, Islet product characteristics and factors related to successful human islet transplantation from the Collaborative Islet Transplant Registry (CITR) 1999-2010, Am J Transplant 14(11) (2014) 2595-606.

[15] N. Niclauss, D. Bosco, P. Morel, S. Demuylder-Mischler, C. Brault, L. Milliat-Guittard, C. Colin, G. Parnaud, Y.D. Muller, L. Giovannoni, R. Meier, C. Toso, L. Badet, P.Y. Benhamou, T. Berney, Influence of donor age on islet isolation and transplantation outcome, Transplantation 91(3) (2011) 360-6.

[16] R. Timpl, J.C. Brown, Supramolecular assembly of basement membranes, Bioessays 18(2) (1996) 123-32.

[17] J.H. Van Deijnen, P.T. Van Suylichem, G.H. Wolters, R. Van Schilfgaarde, Distribution of collagens type I, type III and type $\mathrm{V}$ in the pancreas of rat, dog, pig and man, Cell Tissue Res 277(1) (1994) 115-21. 
[18] S.J. Hughes, A. Clark, P. McShane, H.H. Contractor, D.W. Gray, P.R. Johnson, Characterisation of collagen VI within the islet-exocrine interface of the human pancreas: implications for clinical islet isolation?, Transplantation 81(3) (2006) 423-6.

[19] I. Virtanen, M. Banerjee, J. Palgi, O. Korsgren, A. Lukinius, L.E. Thornell, Y. Kikkawa, K. Sekiguchi, M. Hukkanen, Y.T. Konttinen, T. Otonkoski, Blood vessels of human islets of Langerhans are surrounded by a double basement membrane, Diabetologia 51(7) (2008) 1181-91.

[20] P. Bedossa, G. Lemaigre, J. Bacci, E. Martin, Quantitative estimation of the collagen content in normal and pathologic pancreas tissue, Digestion 44(1) (1989) 7-13.

[21] H.A. Tons, O.T. Terpstra, E. Bouwman, Heterogeneity of human pancreata in perspective of the isolation of the islets of langerhans, Transplant Proc 40(2) (2008) 367-9. [22] G. Loganathan, S. Venugopal, S. Narayanan, B. Tweed, M.A. Goedde, B. Gunaratnam, W.W. Tucker, P. Goli, S. Mokshagundam, R.C. McCarthy, S.K. Williams, M.G. Hughes, A.N. Balamurugan, Improved recovery of human islets from young donor pancreases utilizing increased protease dose to collagenase for digesting peri-islet extracellular matrix, Am J Transplant 19(3) (2019) 831-843.

[23] R.M. Spiers, S.E. Cross, H.L. Brown, P.A. Bateman, R.H. Vaughan, S.J. Hughes, P.R.V. Johnson, Development of a Simple In Vitro Assay to Assess Digestion of the Extracellular Matrix of the Human Pancreas by Collagenase Enzyme Blends, Cell Transplant 27(7) (2018) 1039-1046.

[24] S.E. Cross, R.H. Vaughan, A.J. Willcox, A.J. McBride, A.A. Abraham, B. Han, J.D. Johnson, E. Maillard, P.A. Bateman, R.D. Ramracheya, P. Rorsman, K.E. Kadler, M.J. Dunne, S.J. Hughes, P.R. Johnson, Key matrix proteins within the pancreatic islet basement membrane are differentially digested during human islet isolation, Am J Transplant 17(2) (2017) 451-461.

[25] S.D. Sackett, D.M. Tremmel, F. Ma, A.K. Feeney, R.M. Maguire, M.E. Brown, Y. Zhou, X. Li, C. O'Brien, L. Li, W.J. Burlingham, J.S. Odorico, Extracellular matrix scaffold and hydrogel derived from decellularized and delipidized human pancreas, Sci Rep 8(1) (2018) 10452.

[26] E. Brauchle, J. Kasper, R. Daum, N. Schierbaum, C. Falch, A. Kirschniak, T.E. Schaffer, K. Schenke-Layland, Biomechanical and biomolecular characterization of extracellular matrix structures in human colon carcinomas, Matrix Biol 68-69 (2018) 180-193.

[27] M. Votteler, D.A. Carvajal Berrio, M. Pudlas, H. Walles, U.A. Stock, K. SchenkeLayland, Raman spectroscopy for the non-contact and non-destructive monitoring of collagen damage within tissues, J Biophotonics 5(1) (2012) 47-56. 
[28] A. Daniel, A. Prakasarao, S. Ganesan, Near-infrared Raman spectroscopy for

estimating biochemical changes associated with different pathological conditions of cervix, Spectrochim Acta A Mol Biomol Spectrosc 190 (2018) 409-416.

[29] J. Hilderink, C. Otto, C. Slump, A. Lenferink, M. Engelse, C. van Blitterswijk, E. de Koning, M. Karperien, A. van Apeldoorn, Label-Free Detection of Insulin and Glucagon within Human Islets of Langerhans Using Raman Spectroscopy, Plos One 8(10) (2013). [30] M.B. Albro, M.S. Bergholt, J.P. St-Pierre, A. Vinals Guitart, H.M. Zlotnick, E.G. Evita, M.M. Stevens, Raman spectroscopic imaging for quantification of depth-dependent and local heterogeneities in native and engineered cartilage, NPJ Regen Med 3 (2018) 3.

[31] T.T. Nguyen, C. Gobinet, J. Feru, S. Brassart-Pasco, M. Manfait, O. Piot, Characterization of Type I and IV Collagens by Raman Microspectroscopy: Identification of Spectral Markers of the Dermo-Epidermal Junction, Spectroscopy-an International Journal 27(5-6) (2012) 421-427.

[32] M. Pudlas, E. Brauchle, T.J. Klein, D.W. Hutmacher, K. Schenke-Layland, Non-invasive identification of proteoglycans and chondrocyte differentiation state by Raman microspectroscopy, J Biophotonics 6(2) (2013) 205-11.

[33] M. Votteler, D.A. Carvajal Berrio, M. Pudlas, H. Walles, K. Schenke-Layland, Noncontact, label-free monitoring of cells and extracellular matrix using Raman spectroscopy, $\mathrm{J}$ Vis Exp (63) (2012).

[34] J. Marzi, E. M. Brauchle, K. Schenke-Layland, M. W. Rolle, Non-invasive functional molecular phenotyping of human smooth muscle cells utilized in cardiovascular tissue engineering. Acta biomaterialia, 89 (2019) 193-205.

[35] M. Pudlas, S. Koch, C. Bolwien, S. Thude, N. Jenne, T. Hirth, H. Walles, K. SchenkeLayland, Raman spectroscopy: a noninvasive analysis tool for the discrimination of human skin cells, Tissue Eng Part C Methods 17(10) (2011) 1027-40.

[36] C.J. Frank, R.L. McCreery, D.C. Redd, Raman spectroscopy of normal and diseased human breast tissues, Anal Chem 67(5) (1995) 777-83.

[37] W.T. Cheng, M.T. Liu, H.N. Liu, S.Y. Lin, Micro-Raman spectroscopy used to identify and grade human skin pilomatrixoma, Microsc Res Tech 68(2) (2005) 75-9.

[38] Z. Huang, A. McWilliams, H. Lui, D.I. McLean, S. Lam, H. Zeng, Near-infrared Raman spectroscopy for optical diagnosis of lung cancer, Int J Cancer 107(6) (2003) 1047-52. [39] M. Gniadecka, H.C. Wulf, N.N. Mortensen, O.F. Nielsen, D.H. Christensen, Diagnosis of basal cell carcinoma by Raman spectroscopy, Journal of Raman Spectroscopy 28(2-3) (1997) 125-129.

[40] B.G. Frushour, J.L. Koenig, Raman scattering of collagen, gelatin, and elastin, Biopolymers 14(2) (1975) 379-91. 
[41] E. O Faolain, M.B. Hunter, J.M. Byrne, P. Kelehan, M. McNamara, H.J. Byrne, F.M. Lyng, A study examining the effects of tissue processing on human tissue sections using vibrational spectroscopy, Vibrational Spectroscopy 38(1-2) (2005) 121-127.

[42] R.K. Dukor, Vibrational Spectroscopy in the Detection of Cancer, Handbook of Vibrational Spectroscopy, John Wiley \& Sons, Ltd2006.

[43] K. Gelse, E. Poschl, T. Aigner, Collagens--structure, function, and biosynthesis, Adv Drug Deliv Rev 55(12) (2003) 1531-46.

[44] R. Timpl, H. Rohde, P.G. Robey, S.I. Rennard, J.M. Foidart, G.R. Martin, Laminin--a glycoprotein from basement membranes, J Biol Chem 254(19) (1979) 9933-7.

[45] M. Dendo, H. Maeda, Y. Yamagata, K. Murayama, K. Watanabe, T. Imura, A. Inagaki, Y. Igarashi, Y. Katoh, M. Ebina, K. Fujimori, K. Igarashi, N. Ohuchi, S. Satomi, M. Goto, Synergistic Effect of Neutral Protease and Clostripain on Rat Pancreatic Islet Isolation, Transplantation 99(7) (2015) 1349-55.

[46] M. Shimoda, H. Noguchi, B. Naziruddin, Y. Fujita, D. Chujo, M. Takita, H. Peng, Y. Tamura, G.S. Olsen, K. Sugimoto, T. Itoh, N. Onaca, M.F. Levy, P.A. Grayburn, S. Matsumoto, Improved method of human islet isolation for young donors, Transplant Proc 42(6) (2010) 2024-6.

[47] D.W. Gray, P. McShane, A. Grant, P.J. Morris, A method for isolation of islets of Langerhans from the human pancreas, Diabetes 33(11) (1984) 1055-61.

[48] S. Hughes, D. Gray, A. Clark, P. Johnson, Development of an assay for the detailed study of collagenase-digestion within the islet-exocrine interface of the human pancreas Xenotransplantation 14 (2007) 485-485.

[49] S.E. Cross, S.J. Hughes, C.J. Partridge, A. Clark, D.W. Gray, P.R. Johnson, Collagenase penetrates human pancreatic islets following standard intraductal administration, Transplantation 86(7) (2008) 907-11.

[50] P.M. Angel, S. Comte-Walters, L.E. Ball, K. Talbot, A. Mehta, Brockbank KGM, et al. Mapping Extracellular Matrix Proteins in Formalin-Fixed, Paraffin-Embedded Tissues by MALDI Imaging Mass Spectrometry, Journal of proteome research 17 (2018) 635-46.

[51] N. Stone, C. Kendall, J. Smith, P. Crow, H. Barr, Raman spectroscopy for identification of epithelial cancers, Faraday Discuss 126 (2004) 141-57; discussion 169-83.

[52] C. Krafft, L. Neudert, T. Simat, R. Salzer, Near infrared Raman spectra of human brain lipids, Spectrochim Acta A Mol Biomol Spectrosc 61(7) (2005) 1529-35.

[53] A.J. Ruiz-Chica, M.A. Medina, F. Sanchez-Jimenez, F.J. Ramirez, Characterization by Raman spectroscopy of conformational changes on guanine-cytosine and adenine-thymine oligonucleotides induced by aminooxy analogues of spermidine, Journal of Raman Spectroscopy 35(2) (2004) 93-100. 
[54] J.W. Chan, D.S. Taylor, T. Zwerdling, S.M. Lane, K. Ihara, T. Huser, Micro-Raman spectroscopy detects individual neoplastic and normal hematopoietic cells, Biophysical Journal 90(2) (2006) 648-656.

\section{Figure legends}

\section{Figure 1: Raman spectra for ECM reference standards}

(A) Spectra display the fingerprint region of purified matrix proteins following baseline correction and normalisation $\left(400-1800 \mathrm{~cm}^{-1}\right)$. The four proteins display similar spectra, with a few key variations. Spectral differences are seen in the regions corresponding to (B) Proline and (C) $\mathrm{CH}_{2}$ deformation. (D) General peak assignments for purified ECM proteins. Dashes indicate the absence of a peak.

ECM; Extracellular matrix

Figure 2: Enzyme induced changes in Raman biomolecular fingerprint of ECM proteins Principle component analysis (PCA) was performed on spectra obtained from collagenase and neutral protease treated purified proteins. Protein treatment occurred for 30 and 120 minutes, or overnight. Control samples were heated overnight in HBSS.

(A) Collagen I treatment with enzymes led to separation along PC-3 and (B) the corresponding PC-3 loading highlighting the main peaks responsible for the separation (C) Collagen IV separation was along PC-2 and (D) corresponding PC-2 loading (E) Collagen VI separation occurred along PC-1 and (F) corresponding PC-1 loading (G) Laminins separation occurred along PC-1 and (H) corresponding PC-1 loading.

PC; Principle component

\# Indicates significant difference from control

* Indicates significant difference between the two groups, as indicated by the lines 
Spiers et al.

Donor age significantly influences the Raman spectroscopic biomolecular fingerprint of human pancreatic extracellular matrix proteins following collagenase-based digestion

$\star \star \# \# p<0.01$

$\star \star \star / \# \#$ p $<0.001$

Figure 3: Raman imaging of pancreatic tissue

Multivariate analysis identified three major spectral components. Morphologically the components represented the cytoplasm (component 1; pink), the ECM (component 2; green) and nuclei (component 3; blue).

Scale bar equals $40 \mu \mathrm{m}$

ECM; extracellular matrix

Figure 4: Correlation of Raman imaging and immunofluorescence staining (A-C) Collagen IV immunofluorescent images (D-F) Label-free images of the ECM component identified by multivariate analysis, on the basis of the samples Raman spectra (G-I) Composite images, created by merging the corresponding fluorescent (green) and Raman images (pink), demonstrate a high degree of homogeneity between the protein signature identified by the two methods.

Scale bars equals $40 \mu \mathrm{m}$

ECM; Extracellular matrix

Figure 5: Acquisition of Raman spectra from enzyme treated pancreatic tissue sections (A) Representative immunofluorescent images of control tissue from younger and older donors at 10X, demonstrating identification of islets via positive insulin (red) and collagen IV (green) labelling. (B) Representative focus of Raman laser on islet extracellular matrix, as identified by collagen IV, at $60 \mathrm{X}$, following controlled enzyme induced digestion. The crosses depict the areas analysed for one Raman measurement, obtained from the islet peripheral (white crosses) and the intra-islet region (yellow crosses). A minimum of 40 measurements were collected for each condition, from a minimum of two islets, for each donor. 
Donor age significantly influences the Raman spectroscopic biomolecular fingerprint of human pancreatic extracellular matrix proteins following collagenase-based digestion

Scale bars $100 \mu \mathrm{m}(\mathrm{A}), 30 \mu \mathrm{m}(\mathrm{B})$.

Figure 6: Principle Component Analysis (PCA) of age-related differences in the enzyme induced digestion of the ECM

(A) PCA scores plot demonstrate donor age-related separation in collagenase treated tissues

(B) PC-5 indicates the main differences in the spectra responsible for the separation following collagenase treatment $(\mathbf{C})$ donor age separation of Raman spectra acquired from neutral protease treated tissue was less clear (D) corresponding loading spectra for neutral protease treated tissue (E) PCA scores plot display a good age-related separation for tissue treated with collagenase and neutral protease combined (F) PC-4 indicates main regions of spectral difference following treatment with the enzyme combination

$* p<0.05$

$\star \star \star ~ p<0.001$

ECM; Extracellular matrix

\section{Figure 7: High resolution Raman imaging identifies additional matrix structures}

(A, B) Fluorescent images of islets, identified by collagen IV labelling. (C, D) Multivariate analysis of corresponding higher resolution Raman images identified an additional ECM assigned component (seen in orange; ECM Component 2). The intensity of this component appeared to be higher in the intra-islet region. (E) The corresponding spectra for the two components show clear differences in composition.

Scale bar equals $20 \mu \mathrm{m}$

\section{Figure 8: Detailed analysis of ECM structures within the islet region}

(A) For in depth analysis of the ECM structures present at the islet-exocrine interface, randomly selected single spectra from the high resolution scans were extracted from the peripheral (peri; in blue) and intra- islet (in red) ECM. (B) A PCA was performed showing a 
highly significant difference in PC-3 for the spectral information of the groups. (C) The loadings plot described collagen-related peaks as the main influencing differences of the data sets. (D) Spectral assignments.

Scale bar equals $20 \mu \mathrm{m}$

*** $p<0.001$

ECM; extracellular matrix

PCA; Principle component analysis

\section{Tables}

\begin{tabular}{|l|c|c|c|}
\cline { 2 - 4 } \multicolumn{1}{c|}{} & $\begin{array}{c}\text { Younger Donors } \\
\mathbf{5} \mathbf{3 0} \text { years } \\
(\mathbf{n}=\mathbf{5})\end{array}$ & $\begin{array}{c}\text { Older Donors } \\
\mathbf{5} \mathbf{5 5} \text { years } \\
\mathbf{( n = 5 )}\end{array}$ & $\boldsymbol{p}$ value \\
\hline $\begin{array}{l}\text { Age, y (Mean } \pm \\
\text { SD) }\end{array}$ & $27 \pm 0.5$ & $58 \pm 0.3$ & $<0.001$ \\
\hline $\begin{array}{l}\text { Age, y (Median \& } \\
\text { Range) }\end{array}$ & $26(25-30)$ & $58(56-60)$ & $<0.001$ \\
\hline Gender (M:F) & $4: 1$ & $3: 2$ & 0.49 \\
\hline BMI (Mean \pm SD) & $26.3 \pm 3.9$ & $28.6 \pm 1.9$ & 0.28 \\
\hline $\begin{array}{l}\text { CIT, h (Mean } \pm \\
\text { SD) }\end{array}$ & $6.2 \pm 1.5$ & $6.1 \pm 1.4$ & 0.92 \\
\hline
\end{tabular}

Table 1: Donor and pancreas characteristics

CIT; Cold ischemia time

SD; Standard deviation 


\begin{tabular}{|c|c|c|c|}
\hline Peak $\left[\mathrm{cm}^{-1}\right]$ & $\begin{array}{c}\text { Cytoplasm } \\
\text { (Component 1) }\end{array}$ & $\begin{array}{c}\text { ECM } \\
\text { (Component 2) }\end{array}$ & $\begin{array}{c}\text { Nuceli } \\
\text { (Component 3) }\end{array}$ \\
\hline 784 & & & DNA [51] \\
\hline 854 & & C-C (proline collagen) [40] & \\
\hline 938 & & C-C (collagen backbone) [40] & \\
\hline 1081 & Lipids [52] & & \\
\hline 1098 & & & Phosphate [42] \\
\hline 1123 & $\begin{array}{c}\text { C-N stretch (proteins) } \\
{[38]}\end{array}$ & & \\
\hline 1245 & & amide III [40] & \\
\hline 1251 & & & $\mathrm{NH}_{2} \mathrm{G}, \mathrm{C}[54]$ \\
\hline 1299 & $\begin{array}{c}\mathrm{CH}_{2} \text { deformation (lipids) } \\
{[51]}\end{array}$ & & \\
\hline 1372 & & & DNA bases [54] \\
\hline 1445 & & $\begin{array}{c}\mathrm{CH}_{2} \text { deformation } \\
\text { (proteins/collagen) [40] }\end{array}$ & \\
\hline 1577 & & & $\mathrm{NADH}, \mathrm{G}[53]$ \\
\hline
\end{tabular}

Table 2: Peak assignments for Raman imaging of pancreatic tissue

Molecular assignment of the components identified in Figure 3

NADH; Nicotinamide adenine dinucleotide 